\title{
Scheduling for Video Transmission in LTE Downlink
}

\author{
Princy P. Jayan, Luxy Mathews, Sakuntala S. Pillai \\ Department of Electronics and Communication Engineering Mar Baselios College of Engineering and \\ Technology, Thiruvananthapuram, India
}

\begin{abstract}
Initial developments of cellular networks were intended for voice only applications. Later the need to support several applications and services lead to the evolution of mobile technology. In order to meet the growing user demands and high data rate requirements the fourth generation of mobile communication has adopted a standard called LTE. LTE provides high data rates, flexible bandwidth usage and low latency in networks. Scheduling is a feature employed at the eNodeB to allocate user equipments with available resources in the most efficient way. A scheduling scheme for video transmission is proposed in the downlink. A decision index is introduced in the algorithm which considers the status of the transmission queue and the quality of the channel connecting the eNodeB and the intended user equipment. Higher decision indexed valued user data is scheduled first.
\end{abstract}

Index Terms: 4G, LTE, Video Codec, H.264, Resource Block, Channel Quality, CQI, Scheduling.

\section{Introduction}

Individuals exchange information between them through the process of communication. Communication can be done by any means such as gestures, vocabulary, written scripts and wired or wireless means. Nowadays wireless communication systems play a vital role in revolutionizing the means of information exchange. Mobile Communication categorically falls into the division of wireless communication. It is now one of the fastest means for conveying information between individuals. Mobile technology has evolved from the usage of analog devices to the digital counter parts. From first generation (1G) of mobile communication to the forthcoming fifth generation $(5 \mathrm{G})$ the difference in communication speed and bandwidth flexibility is clearly visible. Amplitude and frequency modulation techniques employed in the zeroth generation of mobile technology gave only a handful of channels for communication. Analog devices were designed for voice only applications in $1 \mathrm{G}$ which gave only low capacity voice channels. Later with digital techniques and multiple access schemes $2 \mathrm{G}$ provided unlimited access to limited channels. Wider range of applications such as voice telephony, video call, mobile TV, GPS (Global Positioning System) and video conferencing were supported by the third generation $(3 \mathrm{G})$ of mobile communications. However with the advent of newer technologies and a hike in user demands, the need for next generation of mobile technology became inevitable. Thus the evolution of fourth generation mobile technology occurred. $4 \mathrm{G}$ is clearly an improvement from its pre-existing technologies in terms of spectral efficiency, data rates and network capacity. LTE is the wireless standard adopted by the fourth generation of mobile technology. The adaptive modulation and coding (AMC) schemes, MIMO antenna technique and multicarrier modulation schemes such as OFDM jointly contributes to the high achievable data rates and spectral efficiency [3].

In LTE systems, scheduling and resource allocation are the two important features adopted to ensure user satisfaction as well as efficient utilization of the available spectrum. Scheduling ensures the proper transmission and reception of the most urgent user data in the queue. Assignment of radio resources to users based on their quality of service $(\mathrm{QoS})$ requirements and quality of the communication links is the main function of the scheduling and resource allocation processes.

\section{Long Term Evolution}

Long Term Evolution (LTE) is the wireless standard intended for the development of fourth generation of mobile communications. It provides high data rates, flexible bandwidth capabilities, lower latency and high capacity for the systems. Flexible bandwidth feature in LTE systems allows the network operators to access the LTE bandwidth ranging from 1.4 MHz to $20 \mathrm{MHz}$. In LTE systems, the base station is called the evolved Node $\mathrm{B}$ or eNodeB (eNB) and the mobile terminal is called the user equipment (UE). In downlink communication, the eNodeB is the transmitter and user equipment is the receiver whereas in the uplink it is the vice versa. A high data rate of $300 \mathrm{Mbps}$ in the downlink and $75 \mathrm{Mbps}$ in uplink is achieved with increased network coverage and higher user mobilities. LTE follows a fully IP based architecture where all the devices are identified by an IP address. LTE system architecture consists of UE, LTE EUTRAN (eNB) and LTE Evolved Packet Core (EPC) as the network elements [1]. They are connected to each other via interfaces Uu, X2 and S1. LTE EPC architecture includes the Mobility Management Entity (MME), Serving Gateway (SGW), and PDN Gateway (PGW), Home 
Subscriber Server (HSS) and Policy Control and Charging Rules Function (PCRF) entities. LTE uses the MIMO and OFDM as its two key technologies. MIMO enables it to improve the system capacity and performance whereas the multicarrier modulation scheme enables unlimited access to the available radio spectrum. OFDMA and SC-FDMA is used by the LTE systems in downlink and uplink respectively [1]. FDD and TDD are the two duplexing modes supported by LTE. Radio resource management techniques adopted ensures satisfactory quality of service (QoS) to the users. Transmission mode selection, precoding, scheduling and resource allocation are the primal processes that take place at the eNodeB.

\section{Scheduling}

A resource element (RE) is comprised of one subcarrier and one OFDM symbol along the frequency and time domain respectively. The subcarrier has a bandwidth of $15 \mathrm{kHz}$. A resource block (RB) is the fundamental scheduling unit. It is formed by many resource elements. It consists of 12 subcarriers along frequency domain and 7 OFDM symbols along the time domain [1]. A time slot is of duration 0.5 millisecond and is formed by 7 OFDM symbols. A resource block pair comprising of 14 OFDM symbols and 12 subcarriers is as shown in Figure 1. It has $(12 \times 15 \mathrm{kHz}) 180 \mathrm{kHz}$ bandwidth and $0.5 \mathrm{~ms}$ duration.

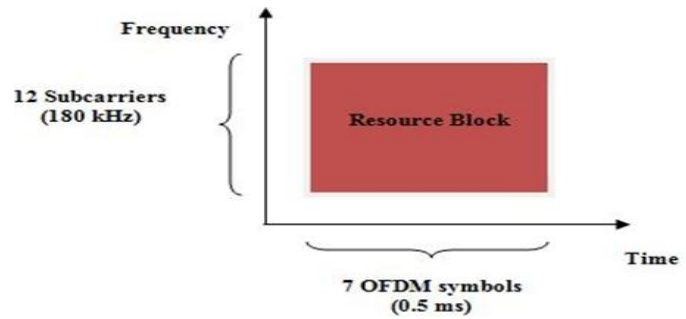

Figure 1: Resource Block

Scheduling is the process of deciding on the number of resources to be given to a user equipment to send or receive data [1]. The scheduling process takes place at the eNodeB. Scheduling in the downlink involves allocating resources to the user equipment by the eNB to receive the data. The process initially starts with the user equipment sending the channel quality indicator (CQI) value and the buffer state report (BSR) to the eNodeB. This information is then used by the eNodeB in allocating the resource blocks and employing the proper modulation and coding scheme.

CQI value indicates the quality of the radio channel for each user on each RB. It is a four bit value ranging from 0 to 15.A higher $\mathrm{CQI}$ value indicates a better channel while a lower value indicates a worse channel for transmission. The CQI value is measured by the UE from the estimated SNR. Corresponding to every CQI value there is a modulation scheme that can be adopted. For higher CQI values higher levels of modulation is adopted as shown in Table I.

\section{Video Compression Technique}

Video is a sequence of images or frames [5]. A number of frames are required to form a video and such videos will be large in size. A larger storage space becomes necessary to store large sized videos. Moreover the bandwidth required for transmission of such uncompressed videos is also huge. Such requirements of larger storage space and huge bandwidth are not reasonable. Hence it is required to compress the uncompressed videos in order to fit into the available storage space and bandwidth. For this purpose video compression techniques are employed. There are many video compression techniques available today. This includes MPEG-2, MPEG-4, H.261, H.263 and H.264. MPEG-2 was mainly used in DVD compression whereas H.264 has its application in low bandwidth mobile and IP based video telecommunications networks.

Table I: CQI Table

\begin{tabular}{|c|c|}
\hline CQI Value & Modulation Scheme \\
\hline 0 & - \\
\hline 1 & QPSK \\
\hline 2 & QPSK \\
\hline 3 & QPSK \\
\hline 4 & QPSK \\
\hline 5 & QPSK \\
\hline 6 & QPSK \\
\hline 7 & 16 QAM \\
\hline 8 & 16 QAM \\
\hline 9 & 16 QAM \\
\hline 10 & 64 QAM \\
\hline 11 & 64 QAM \\
\hline 12 & 64 QAM \\
\hline 13 & 64 QAM \\
\hline 14 & 64 QAM \\
\hline 15 & 64 QAM \\
\hline
\end{tabular}


H.264 video codec is a combination of the encoder and decoder. There mainly two types of frames involved in the technique. They are I and P frames respectively. An independant set of frames in a video is called the Group of Pictures (GOP). I frame is the main frame in a group of pictures (GOP) and is independant of the other frames in the group. $\mathrm{P}$ frame is the predicted frame and it depends on one or more previous frames. The working of the H.264 video compression technique is illustrated as a block diagram in Figure 2.

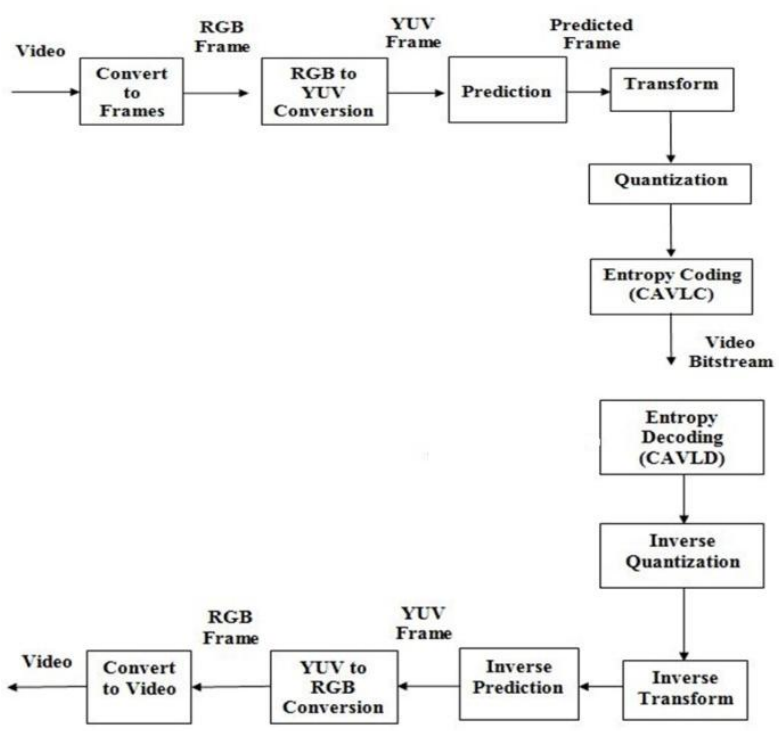

Figure 2: H.264 Video Codec

The video is initially converted to individual frames in RGB format. Since the video codec works on YUV format images, the frames are converted from RGB to YUV format. Prediction block performs intra prediction on I frames and inter prediction on $\mathrm{P}$ frames. The output of the prediction block is then transformed, quantized and entropy coded to obtain a compressed video bitstream $[5,7,8]$. At the decoder the reverse process occurs. Video is reconstructed back from the video bitstream through the process of entropy decoding, inverse quantization, inverse transform and inverse prediction.

\section{A. System Model}

\section{Proposed Method}

The system model consists of video encoder and decoder operating on $\mathrm{N}_{\mathrm{u}}$ number of video sequences, an eNodeB, a downlink scheduler at the eNodeB, CQI feedback from user equipments to eNodeB, an LTE channel and $N_{u}$ user equipments. This is illustrated in Figure 3. Consider $N_{u}$ number of video sequences to be transmitted to $\mathrm{N}_{\mathrm{u}}$ number of UE. Each video is encoded to a compressed video bitstream and stored in the buffer at eNodeB. The downlink scheduler at the eNodeB schedules each video bitstream $\mathrm{N}_{\mathrm{x}}$ according to their priority for transmission through the LTE channel. At the receiver end, the UE to receive the corresponding video receives the video bitstream $\mathrm{N}_{\mathrm{x}}$ and decodes it to obtain the video.

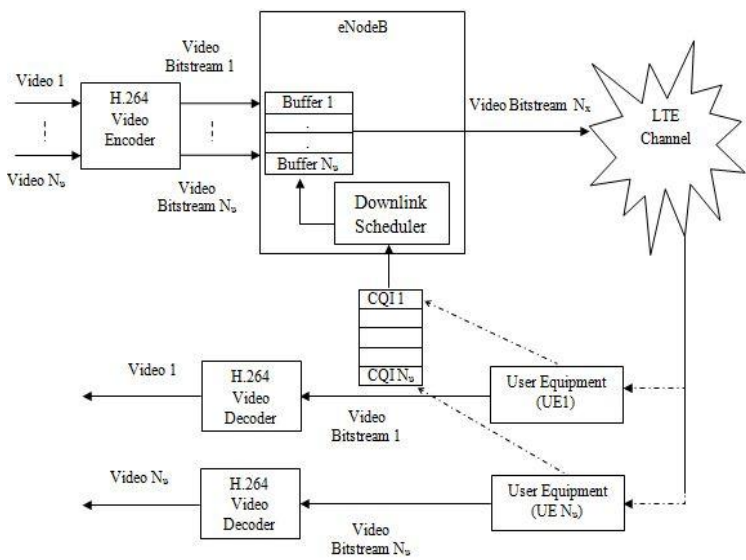

Figure 3: System Model 


\section{B. Scheduling Algorithm}

A downlink scheduling scheme is employed which considers the amount of video data to be transmitted to the UE and the quality of the channel in the downlink. Figure 4 shows the flow chart implementation of the downlink scheduler algorithm. The video bitstream to be transmitted to different UE and the CQI value of each UE over each RB is considered.

The scheduler works in parallel by prioritizing the users based on the amount of video data to be received by the UE and finding the best channel quality value of each UE from the available CQI values. User prioritization means giving priority to the UE based on their amount of video data to be received. The video bit length of each video bitstream is computed and arranged in the decreasing order of their bit length. The UE to receive highest video bit length is given the highest priority and the user to receive the smallest video bit length is given the least priority. The best CQI value among the CQI values over each RB of each UE is also computed.

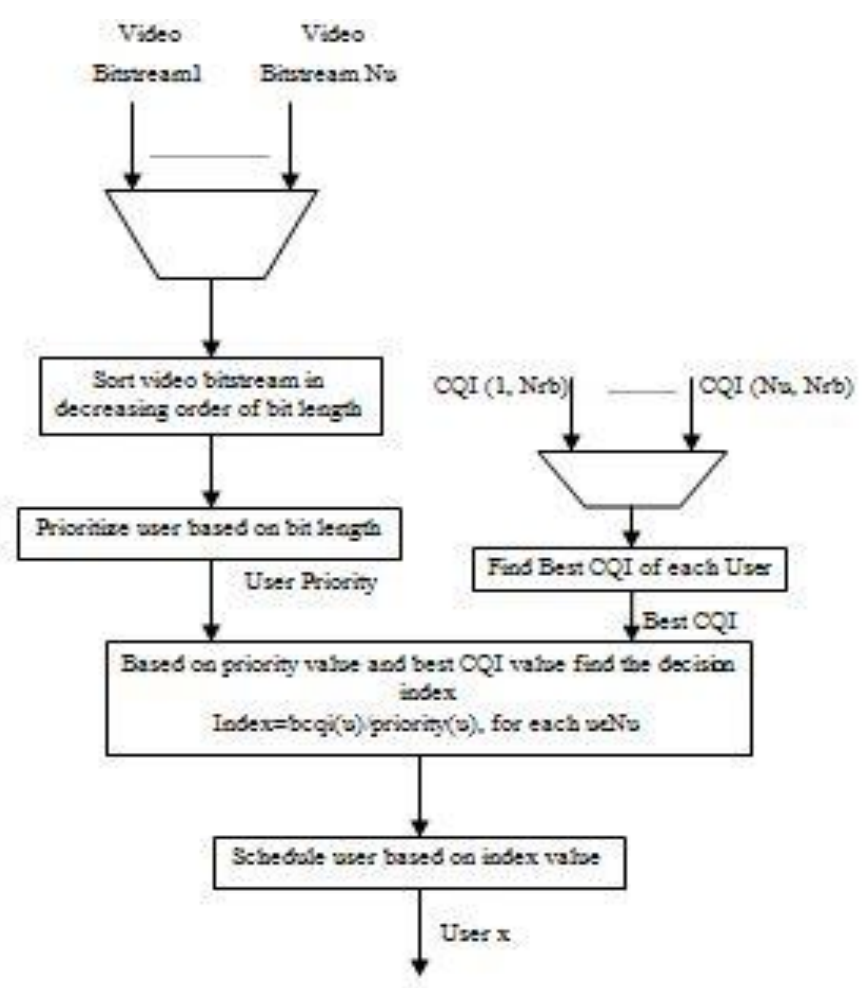

Figure 4: Flow chart of downlink scheduling algorithm

Based on the priority value of user equipment and its channel quality fed back to the transmitter a decision index is calculated. Decision index is the ratio of best CQI value and the priority value of each UE. Thus the transmitter selects the best user among the recipient user equipment according to the user priority and quality of channel between the transmitting eNodeB and the receiving user equipment. The algorithm favors the user with higher user priority and best channel quality i.e. the user with the highest decision index is first scheduled and allocated with resource blocks for transmission.

\section{Simulation Results}

A video encoder was set up in MATLAB 2014a simulation platform to obtain the compressed video bitstream. Two different videos with different GOP sizes were given to the video encoder to obtain video bitstreams of different video bit length for 10 users in the downlink. From the Figure 5(a) it is clear that 5 users receive video 1 of different video bit length and other 5 users receive video 2 of different video bit length. The video bit length for 10 users using two different videos are considered in Figure 5(b).

To obtain the user priority the video bit length is arranged in the decreasing order of their bit size. It is evident from Figure 6 that the user 5 is going to receive the highest bit size video bitstream and user 6 is going to receive the least bit size video bitstream. Therefore the priority of user 5 is 1 and that of user 6 is 10 . 

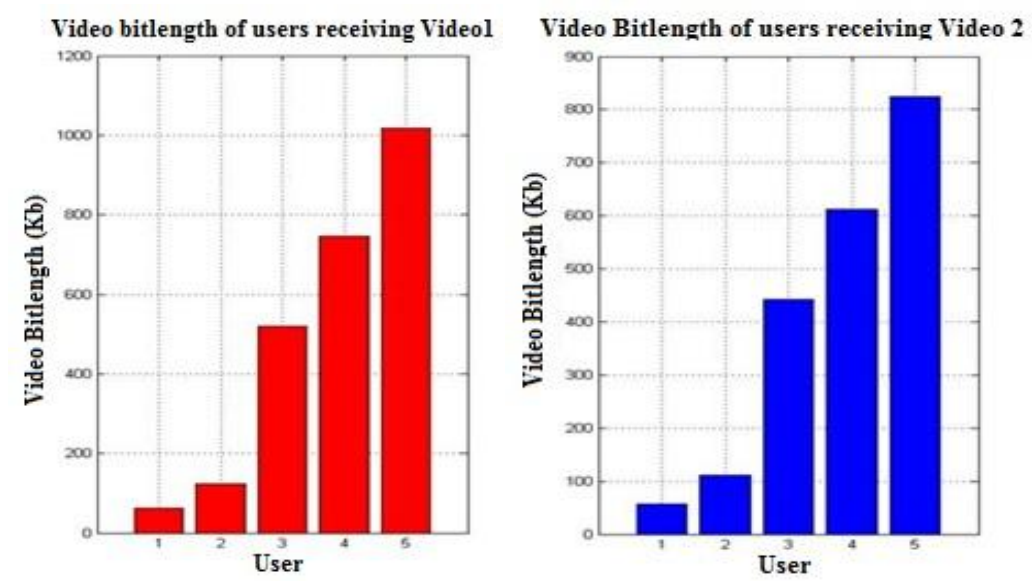

Figure 5 (a): Video bitstream length of users receiving video 1 and video 2

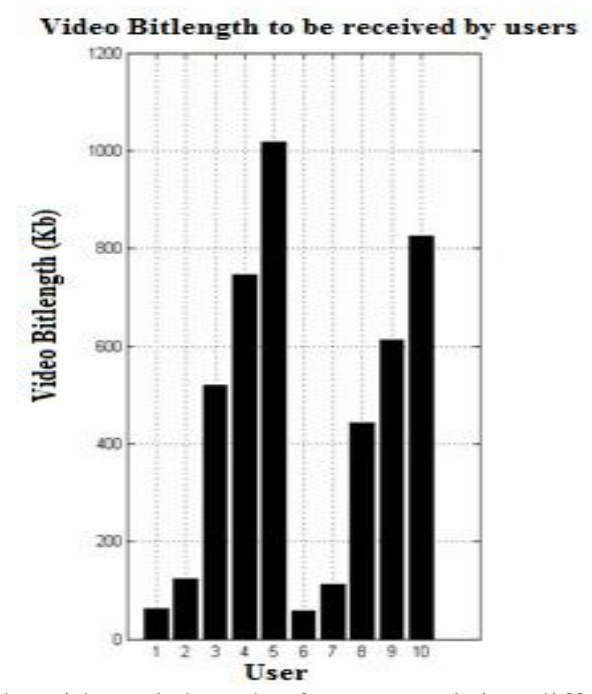

Figure 5(b): Video Bit length of users receiving different videos
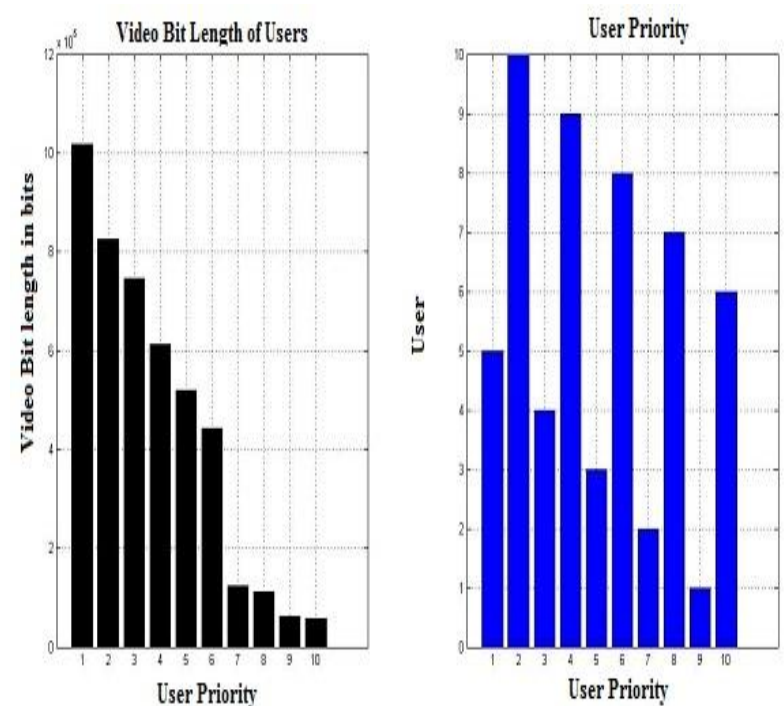

Figure 6: User Prioritization

The channel quality is measured in terms of the CQI value. Its value ranges from 1 to 15 . A CQI value of 15 indicates a channel with better quality. As the value of CQI decreases, the quality of the channel also degrades. The CQI value for 10 users over 6 resource blocks for an LTE system bandwidth of $1.4 \mathrm{MHz}$ is as shown in Figure 7. 


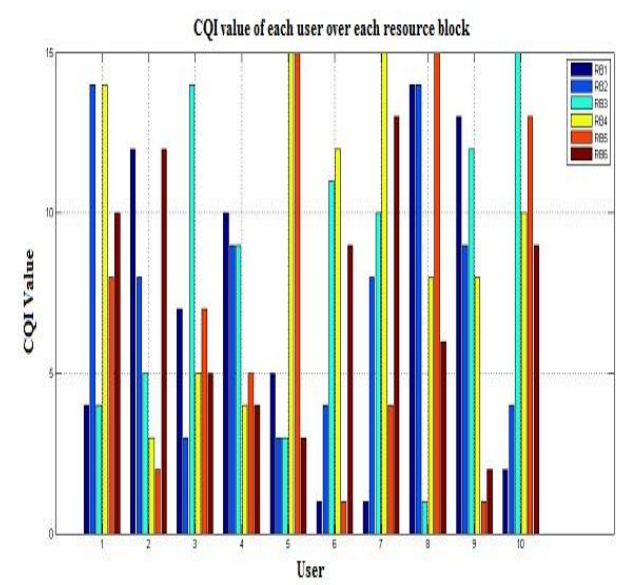

Figure 7: CQI values of each user over every resource block

From among the CQI values over the six resource blocks, the best CQI value is selected for each user. The best CQI value of each user is shown in Figure 8. User 1 and User 3 are having a best CQI value of 14. User 2 and User 6 are having a best CQI value of 12. User 5, 7, 8 and 10 have the best CQI value of 15. User 9 has a maximum CQI value of 13 and User 4 has a maximum CQI value of 10 .

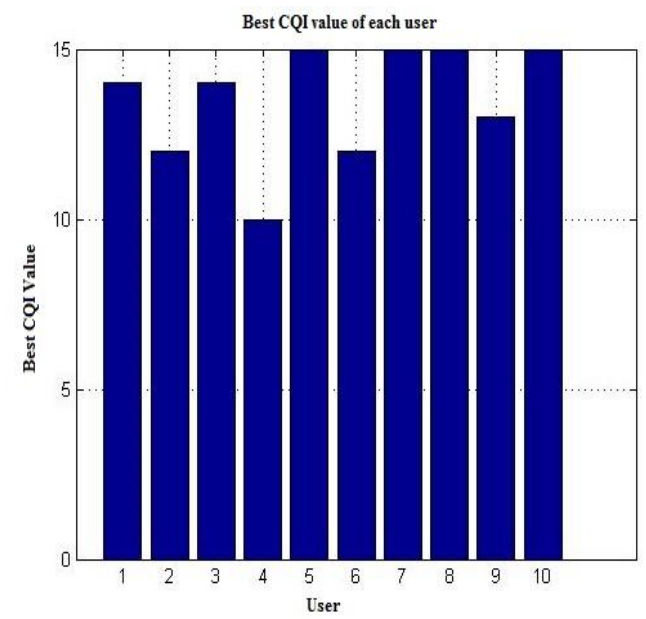

Figure 8: Best CQI value of each user

Using the priority value and the best CQI value of each user, a decision index is calculated and is obtained as shown in Figure 9. The highest decision index is for user 5. Hence user 5 will first be scheduled and allocated with resource blocks. Resource blocks with CQI values equal to or nearer to the best CQI value are considered and allocated to each user.
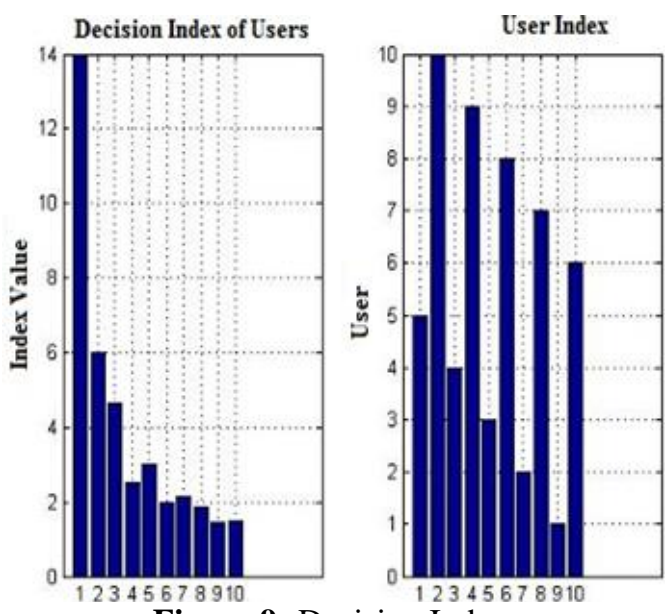

Figure 9: Decision Index 
It is evident from Figure 9 that the decision index of User 3 is more than User 9. So resources will first be allocated to User 3 and then User 9 even though User 9 is having higher priority than User 3 . This difference is occurring because the channel quality of User 3 is better than that of User 9.

Resource block allocation to the users is done based on their decision index value. Scheduling and resource allocation priority is User 5 first and User 10, 4, 3, 9, 2, 8, 7, 1 and 6 follows. The resource allocation of the users and user index is given in Figure 10.
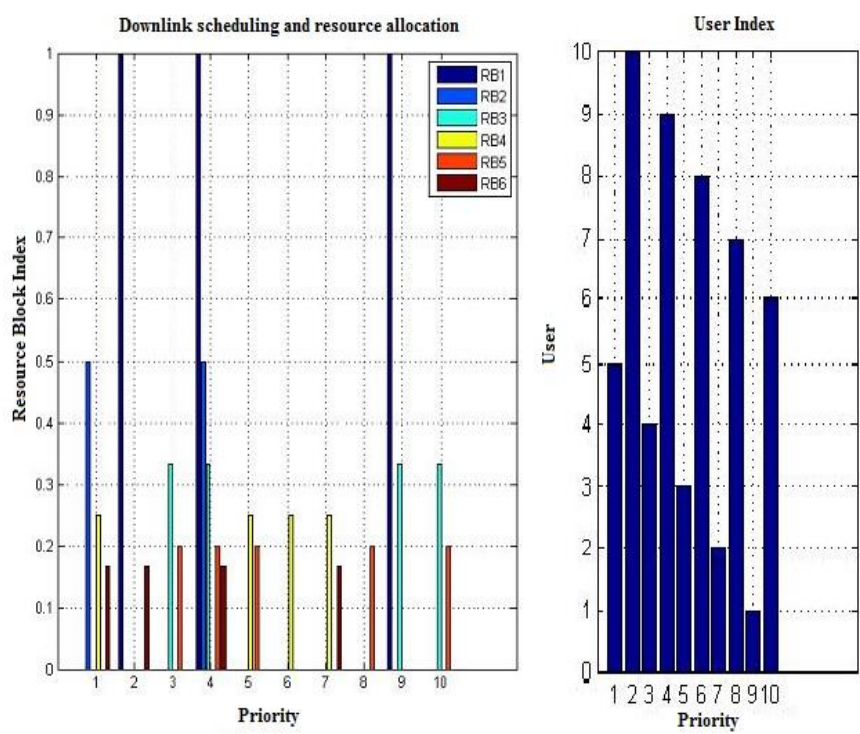

Figure 10: Resource Allocation and Scheduling

User 5 is given resource blocks RB2, RB4 and RB6 having maximum CQI values. User 8 is given only one resource block RB4. User 9 is provided with RB1, RB2, RB3, RB5 and RB6 except RB4 i.e. five out of six resource blocks are allocated to User 9 for transmission in one TTI (Transmission Time Interval) of 1 millisecond. Depending upon the decision index User 3 will be first allocated with resources and then User 9.

\section{Conclusion}

LTE is a wireless standard adopted by next generation mobile technology. Scheduling is an important feature in LTE. Downlink scheduling involves the allocation of resources by eNodeB to the user equipments. This can be done based on the status of the transmission queue, channel quality information and QoS requirements of the users [3]. The downlink scheduler employed takes into account both the transmission queue status and the channel quality of the user equipment. A decision index is calculated considering the aforementioned two factors. The user equipment with the highest index is scheduled first and is allocated with available resources.

\section{References}

[1] Qian Liu, and Chang Wen Chen, "Smart Downlink Scheduling for Multimedia Streaming over LTE Networks with Hard HandOff”, IEEE Transactions on Circuits and Systems for Video Technology, 2015.

[2] Mohammed Alhadi Abduljalil, "Resource Scheduling Algorithms in Long Term Evolution (LTE)", Journal of Electronics and Communication Engineering Volume 9, Issue 6, PP 50-53, Nov - Dec. 2014.

[3] F. Capozzi, G. Piro, L.A. Grieco, G. Boggia, and P. Camarda, "Downlink Packet Scheduling in LTE Cellular Networks: Key Design Issues and a Survey", IEEE Communications Surveys \& Tutorials, Vol. 15, No. 2, Second Quarter 2013.

[4] A. S. Sravani and K. Jagadeesh Babu, "Implementation of Scheduling Algorithms for LTE Downlink", International Journal of Advanced Electrical and Electronics Engineering (IJAEEE), Volume-2, Issue -6, ) ISSN (Print): 2278 - 8948, 2013.

[5] Manjanaik N, Dr. Manjunatha R, “ Development of Efficient Intra Frame Coding in Advanced Video Standard Using Horizontal Prediction Mode", International Journal of Emerging Technology and Advanced Engineering, Volume 3, Issue 2, February 2013.

[6] Jean Thierry Stephen Avocanh, Marwen Abdennebi, Jalel Ben-Othman , "A New Two-Level Scheduling Algorithm for the Downlink of LTE Networks", Wireless Networking Symposium, Globecom 2013.

[7] Jian Wen Chen, Chao-Yang Kao and Youn Long Lin, "Introduction to H.264 Advanced Video Coding", Asia and South pacific conference on design automation, January, 2006.

[8] Henrique S. Malvar, Antti Hallapuro, Marta Karczewicz, and Louis Kerofsky, "Low-Complexity Transform and Quantization in H.264/AVC", IEEE Transactions On Circuits And Systems For Video Technology, Vol. 13, No. 7, July 2003. 